\section{Promoción de salud y actividad física en Chile: política prioritaria}

\author{
Judith Salinas ${ }^{1}$ y Fernando Vio ${ }^{2}$
}

Palabras clave: actividad física, estilo de vida, Chile.

\footnotetext{
1 Departamento de Promoción de la Salud, Ministerio de Salud de Chile. La correspondencia debe enviarse a Judith Salinas, Mac Iver 541, Of. 521, Santiago, Chile. Correo electrónico: jsalinas@minsal.cl

2 Instituto de Nutrición y Tecnología de los Alimentos, Universidad de Chile, Santiago, Chile. Correo electrónico: fvio@inta.cl
}

Chile ha experimentado cambios muy rápidos en su perfil epidemiológico y nutricional. De una situación epidemiológica en la que predominaban las enfermedades infecciosas y los problemas maternoinfantiles en la década de 1960, pasó en unos 20 años a un perfil epidemiológico con un predominio de enfermedades crónicas no transmisibles, accidentes y problemas de salud mental. Los cambios nutricionales fueron también muy rápidos y la alta prevalencia de desnutrición, característica del período 1960-1970, se pudo reducir a finales de los años ochenta. Este cambio cualitativo fue el resultado de políticas integrales que promovían la entrega de alimentos a todos los beneficiarios de los sistemas de salud (prevención primaria) durante los controles de salud materna e infantil; a las madres y niños en riesgo de desnutrición o con desnutrición leve o moderada (prevención secundaria); y a los pacientes con desnutrición grave hospitalizados en centros especializados (rehabilitación). En la década de 1980 en Chile se observaban simultáneamente casos de obesidad y de desnutrición, y una década después se constató el predominio de la obesidad y de las hiperlipidemias, situación que se mantiene hasta el presente (1). Entre los factores determinantes de esta transición se encuentran los cambios en la dieta y la disminución de la actividad física en la población (2).

Según el censo de 2000 (3), Chile es un país con un $86 \%$ de población urbana, lo cual ha permitido mejorar el acceso a agua potable, alcantarillado, educación y servicios de salud. No obstante, algunos aspectos de la calidad de la vida se han deteriorado, especialmente en los sectores más pobres de la población. Entre las consecuencias negativas de la urbanización se encuentran la alimentación inadecuada, el sedentarismo, el consumo excesivo de tabaco, alcohol y drogas, algunos problemas medioambientales - como la contaminación del aire en las grandes ciudades y el deterioro de los espacios de recreo- - y el aumento de la delincuencia, la violencia y la inseguridad personal (4). Si bien Chile duplicó su ingreso per cápita durante la década de 1990, esto no se tradujo necesariamente en una vida de mejor calidad. De hecho, gran parte de ese incremento en los ingresos se destinó al consumo de comida de alto contenido graso y de azúcar refinada -conocida como "comida chatarra" - y de bebidas gaseosas, así como a la compra de televisores, equipos electrodomésticos y automóviles, con lo que disminuyó la actividad física; además, se incre- 
mentó el consumo de tabaco y alcohol (5). Como consecuencia de esa dieta inadecuada y del sedentarismo se produjo un aumento abrupto de la obesidad en la población, especialmente entre los niños, adolescentes y mujeres en edad fértil (2).

Cada vez hay más indicios del efecto benéfico que tiene la actividad física para la salud y la calidad de la vida de las personas, especialmente por su relación con la obesidad. En Inglaterra se demostró que, a pesar de la disminución en la ingesta de grasas y calorías observada desde la década de 1970, la obesidad aumentó significativamente a partir de 1980, fenómeno que se correlacionó con el aumento del número de automóviles y equipos electrodomésticos, las horas de ocio frente al televisor y la disminución de la actividad física (6). También se ha demostrado que un estilo de vida activo previene las complicaciones de la obesidad, aun cuando no las elimina (7).

Desde la década de 1980, las encuestas - efectuadas con metodologías diversas- muestran un alto nivel de sedentarismo en Chile, el cual aumenta a medida que se incrementa la obesidad en la población. El número de automóviles en el país ha aumentado de 300000 en 1970 a 2200000 en 2002, y cada año se introducen en el mercado nacional unos 100000 vehículos más. De manera similar, en Chile hay más de 2300000 televisores a color distribuidos en hogares de todos los niveles socioeconómicos, lo que ha llevado a que cada persona pase, en promedio, de 2 a 3 horas diarias frente al televisor los días laborales y de 4 a 5 horas durante los fines de semana, independientemente de la edad (8). Por otra parte, a medida que aumenta la población de los asentamientos urbanos, disminuyen las instalaciones donde realizar actividades físicas: hay menos espacios libres para llevarlas a cabo y el aumento de la delincuencia y la inseguridad en las ciudades hace que los niños prefieran quedarse en casa viendo televisión o entretenidos con juegos electrónicos. Además, las horas de actividad física en los jardines infantiles y escuelas se han reducido al mínimo. Para los adultos, el horario laboral se ha prolongado y en Santiago de Chile, por ejemplo, se trabaja 50,6 horas semanales como promedio, es decir, más de 10 horas diarias. Si a ello se agregan el tiempo que lleva ir y venir del trabajo (1-2 horas diarias) y las 2 a 3 horas diarias dedicadas a ver televisión, prácticamente no queda tiempo libre para realizar actividades físicas.

Dos encuestas sobre los factores de riesgo de enfermedades crónicas no transmisibles realizadas en la población mayor de 15 años de la Región Metropolitana de Santiago en 1988 y 1992 demostraron que $60 \%$ de los hombres y $80 \%$ de las mujeres realizaban menos de 15 minutos de actividad física continua dos veces por semana $(9,10)$. Un es- tudio anterior realizado en 1997 en una muestra representativa de la población mayor de 25 años de Valparaíso demostró que $93 \%$ de las mujeres no realizaban ningún tipo de actividad física en su tiempo libre y que esta cifra ascendía a $97 \%$ en mujeres de bajo nivel socioeconómico (11).

Dos encuestas sobre actividad física se efectuaron en el año 2000 con la misma metodología y resultados similares: la Encuesta de Caracterización Socioeconómica (CASEN), efectuada en 20000 hogares en el mes de mayo (12), y la Encuesta de Calidad de Vida y Salud 2000 (ECVS), efectuada en diciembre de ese año y que abarcó 6000 hogares de zonas urbanas y rurales de diferentes regiones del país (13). La pregunta en ambas encuestas fue “¿Cuántas veces ha practicado deporte o alguna actividad física (en sesiones de no menos de 30 minutos)?", con 4 respuestas posibles: 3 ó más veces por semana, 1 ó 2 veces por semana, menos de 1 vez por semana y ninguna. El porcentaje de la población que no realizaba actividad física alguna fue de $71 \%$ (CASEN) y 73\% (ECVS), y el de los que realizaban menos de 30 minutos tres veces por semana, considerados sedentarios, fue de $90,6 \%$ y $91 \%$, respectivamente. En ambas encuestas el sedentarismo fue mayor en las mujeres y en las personas con menor nivel educacional (figura 1).

\section{POLÍTICAS DE PROMOCIÓN DE LA SALUD}

El cambio en el perfil epidemiológico y la situación de los factores de riesgo descrita anteriormente hicieron necesario readecuar las políticas y prioridades de salud en el país. El Ministerio de Salud inició en 1998 el Plan Nacional de Promoción de la Salud $(14,15)$, con metas propuestas para el año 2000 en una primera fase, las que luego se ampliaron con vistas al 2010. Simultáneamente se creó el Consejo Nacional para la Promoción de Salud o VIDA CHILE, organismo intersectorial integrado por 28 instituciones nacionales. ${ }^{3}$ Este consejo está encargado de elaborar las políticas de promoción de salud en el país, coordinar los esfuerzos de las insti-

\footnotetext{
VIDA CHILE está integrado por: Asociación Chilena de Municipalidades, Cámara Nacional de Comercio y Servicios de Chile, Carabineros de Chile, Comisión Nacional del Medio Ambiente, Consejo Nacional para el Control de Estupefacientes, Cruz Roja Chilena, Fondo de Solidaridad e Inversión Social, Fundación Integra, Instituto Nacional de Deportes (Chile Deportes), Instituto Nacional de la Juventud, Instituto de Nutrición y Tecnología de los Alimentos de la Universidad de Chile, Junta Nacional de Auxilio Escolar y Becas, Junta Nacional de Jardines Infantiles. También lo integran los ministerios siguientes: Agricultura, Defensa, Educación, Interior, Planificación y Cooperación, Salud, Secretaría General de Gobierno, Secretaría General de la Presidencia, Servicio Nacional de la Mujer Trabajo y Previsión Social, Vivienda, Urbanismos y Bienes Nacionales; así como la Organización Panamericana de la Salud, el Servicio Nacional del Adulto Mayor, Servicio Nacional del Consumidor y la Sociedad Chilena de Enfermedades Respiratorias.
} 
FIGURA 1. Prevalencia de sedentarismo en la población. Chile, 2000
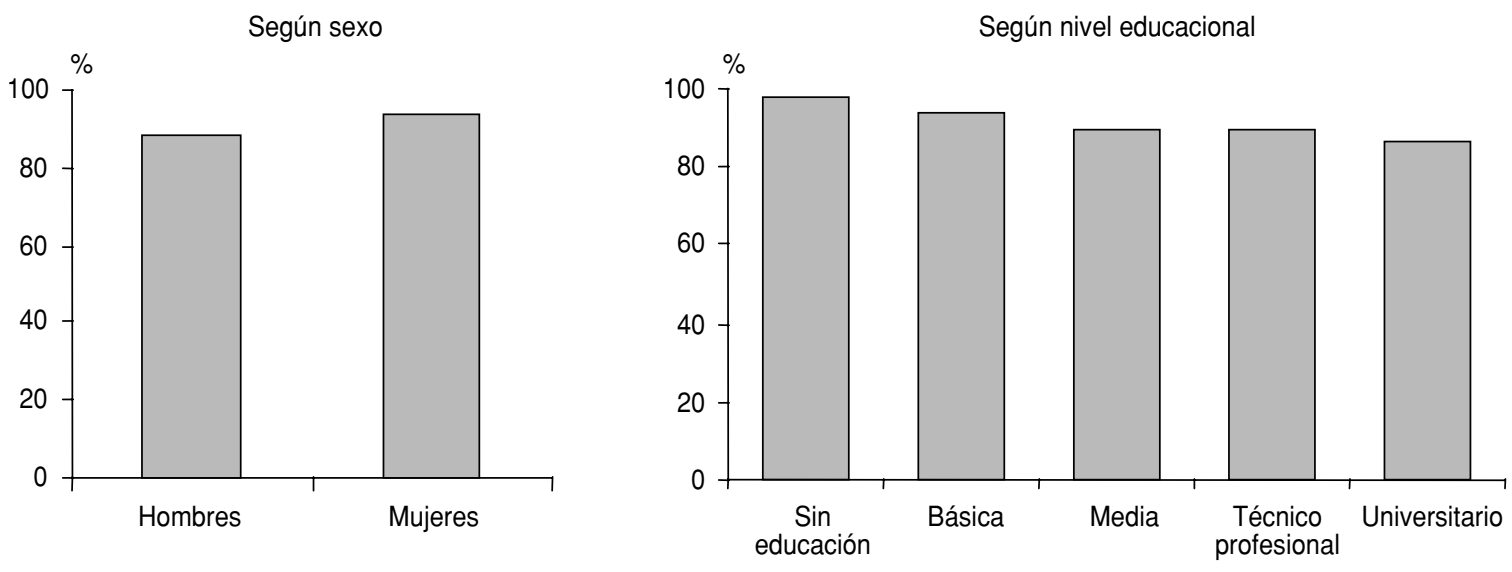

Fuente: Datos de la Encuesta de Calidad de Vida (13).

tuciones participantes, asesorar a los ministerios y a los gobiernos regionales y locales en los temas de promoción, y apoyar la implementación de los planes de promoción de la salud $(16,17)$. VIDA CHILE está organizado en las 13 regiones del país y en 308 de las 341 comunas, las que cuentan con sus consejos VIDA CHILE y planes comunales. Entre las funciones de estos consejos comunales está la de elaborar planes de acción lo más participativamente posible, a manera de garantizar las prioridades locales y su implementación adecuada. Según la ECVS, $45 \%$ de la población chilena participa en alguna organización social, principalmente de tipo religioso, deportivo y territorial (incluidos las juntas de vecinos y los centros de padres y apoderados).

En octubre de 1999 se llevó a cabo el Primer Congreso Chileno de Promoción de Salud, en el que se suscribió el Acta de Huechuraba, denominada "Por un país saludable". Esta acta fue suscrita por autoridades políticas y legislativas, representantes de sectores públicos y privados, científicos y artísticos, comunitarios y sociales, así como por los 1300 asistentes del congreso (18). En ella se reconoce el trabajo ya iniciado y se recoge el consenso de los actores de la sociedad chilena en torno a la promoción de la salud: mantener al individuo sano y mejorar la calidad de la vida de la población. Por todo lo anterior, el Plan Nacional de Promoción de Salud y VIDA CHILE seleccionaron cinco temas prioritarios para las políticas de promoción de la salud: la alimentación, la actividad física, la lucha contra el tabaquismo, los factores psicosociales y los ambientales. Para cada uno de estos temas prioritarios se establecieron metas con vistas al año 2010 (19-21) (cuadro 1) y estrategias de acción (cuadro 2).

\section{Estrategias de actividad física para la promoción de la salud}

Se espera disminuir la prevalencia de sedentarismo en la población de 15 años o más en 7 puntos porcentuales para el año 2010 (de 91 a 84\%). Los objetivos intermedios para cumplir esta meta son la elaboración y difusión de recomendaciones de actividad física para la población chilena, la realización de programas de información y educación para el fomento de una vida activa, la ampliación de las oportunidades para la práctica de actividades físicas y la reducción de las barreras existentes, tales como la falta de espacios y la inseguridad, entre otras.

Las estrategias adoptadas tienen carácter intersectorial y participativo y se llevan a cabo mediante procedimientos educativos y comunitarios destinados a estimular la responsabilidad ciudadana. La implementación de estas estrategias es principalmente responsabilidad del gobierno local mediante los planes comunales de promoción. A su vez, los ministerios son los responsables directos de las estrategias de carácter nacional, como las campañas mediáticas, la formación de recursos humanos y la creación de marcos legislativos y políticos saludables. A continuación se presentan las estrategias más importantes destinadas a disminuir el sedentarismo en Chile, con una reseña de los adelantos logrados mediante cada una de ellas.

Guías de vida activa para la población chilena. En el año 2001 se inició un trabajo conjunto entre representantes de los Ministerios de Salud y de Educación, el Instituto Nacional de Deportes y las universidades, dirigido a elaborar guías para la vida activa de la población chilena. 
CUADRO 1. Metas nacionales en el área de la promoción de salud para los años 2000 y 2010. Chile

\begin{tabular}{|c|c|c|c|c|}
\hline $\begin{array}{l}\text { Temas } \\
\text { prioritarios }\end{array}$ & Metas & Indicador & $2000(\%)$ & $2010(\%)$ \\
\hline \multirow[t]{3}{*}{ Obesidad } & $\begin{array}{l}\text { Disminuir en } 3 \% \text { la prevalencia de } \\
\text { obesidad en preescolares }\end{array}$ & Obesidad en preescolara & $10^{b}$ & 7 \\
\hline & $\begin{array}{l}\text { Disminuir en } 4 \% \text { la prevalencia de } \\
\text { obesidad en } 1^{\circ} \text { básico }\end{array}$ & Obesidad en primero básico a & $16^{c}$ & 12 \\
\hline & $\begin{array}{l}\text { Disminuir en } 4 \% \text { la prevalencia de } \\
\text { obesidad en embarazadas }\end{array}$ & Obesidad en embarazadas & $32^{d}$ & 28 \\
\hline Sedentarismo & $\begin{array}{l}\text { Disminuir en } 7 \% \text { la prevalencia de } \\
\text { sedentarismo en la población mayor } \\
\text { de } 15 \text { años }\end{array}$ & $\begin{array}{l}\text { Sedentarismo en mayores de } \\
15 \text { años }\end{array}$ & $91^{\mathrm{e}}$ & 84 \\
\hline \multirow[t]{3}{*}{ Tabaquismo } & $\begin{array}{l}\text { Disminuir en } 7 \% \text { la prevalencia de } \\
\text { tabaquismo en escolares de octavo } \\
\text { básico }\end{array}$ & $\begin{array}{l}\text { Consumo de tabaco en octavo } \\
\text { básico }\end{array}$ & $27^{\dagger}$ & 20 \\
\hline & $\begin{array}{l}\text { Disminuir en } 5 \% \text { la prevalencia de } \\
\text { tabaquismo en mujeres en edad fértil }\end{array}$ & $\begin{array}{l}\text { Consumo de tabaco en mujeres } \\
\text { en edad fértil }\end{array}$ & $45^{\mathrm{e}}$ & 40 \\
\hline & $\begin{array}{l}\text { Disminuir en } 10 \% \text { la prevalencia de } \\
\text { tabaquismo en la población general }\end{array}$ & $\begin{array}{l}\text { Consumo de tabaco en la } \\
\text { población general }\end{array}$ & $40^{e}$ & 30 \\
\hline Participación & $\begin{array}{l}\text { Aumentar en } 6 \% \text { la participación } \\
\text { ciudadana en organizaciones sociales } \\
\text { de salud }\end{array}$ & $\begin{array}{l}\text { Personas en organizaciones } \\
\text { sociales vinculadas a la salud }\end{array}$ & $4^{e}$ & 10 \\
\hline \multirow[t]{3}{*}{$\begin{array}{l}\text { Espacios } \\
\text { saludables }\end{array}$} & $\begin{array}{l}\text { Recuperar lugares públicos para la vida } \\
\text { saludable }\end{array}$ & $\begin{array}{l}\text { Plazas VIDA CHILE habilitadas } \\
\text { (al menos una plaza por } \\
\text { comuna) }\end{array}$ & - & $\begin{array}{l}100 \% \text { de las } \\
\text { comunas }\end{array}$ \\
\hline & $\begin{array}{l}\text { Acreditar establecimientos de educación } \\
\text { como promotores de salud }\end{array}$ & $\begin{array}{l}\text { Establecimientos de educación } \\
\text { acreditados (preescolar, } \\
\text { escolar, media) }\end{array}$ & - & $\begin{array}{l}50 \% \text { de los } \\
\text { establecimientos } \\
\text { educacionales }\end{array}$ \\
\hline & Acreditar centros de trabajo saludables & Centros de trabajo acreditados & - & $\begin{array}{l}\text { Las metas } \\
\text { porcentuales se } \\
\text { establecen por } \\
\text { cada región }\end{array}$ \\
\hline
\end{tabular}

a Definida como una relación peso:talla mayor de 2 desviaciones estándar.

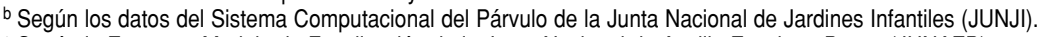

c Según la Encuesta Modelo de Focalización de la Junta Nacional de Auxilio Escolar y Becas (JUNAEB).

${ }^{d}$ Según los datos del Departamento de Informática del Ministerio de Salud. La obesidad en la embarazada se determina según Rosso-Mardones (22).

e Según la Encuesta Nacional de Calidad de Vida del Ministerio de Salud y el Instituto Nacional de Estadísticas (INE).

† Según los datos del Estudio Nacional de Consumo de Drogas de la Comisión Nacional de Control de Estupefacientes (CONACE).

CUADRO 2. Prioridades y estrategias nacionales para la promoción de la salud. Chile, 2000-2010

\begin{tabular}{|c|c|c|}
\hline Prioridades sanitarias & Condicionantes de la salud & Estrategias \\
\hline Enfermedades cardiovasculares & Alimentación & $\begin{array}{l}\text { Nacionales: } \\
\text { - Comunicación }\end{array}$ \\
\hline Salud mental & Actividad física & $\begin{array}{l}\text { - Educación y desarrollo de recursos humanos } \\
\text { - Participación y asociación }\end{array}$ \\
\hline Accidentes & Tabaquismo & $\begin{array}{l}\text { - Reorientación de servicios de salud } \\
\text { - Medidas reguladoras }\end{array}$ \\
\hline Cáncer & $\begin{array}{l}\text { Factores protectores psicosociales } \\
\text { Factores protectores del ambiente }\end{array}$ & $\begin{array}{l}\text { Locales: } \\
\text { - Espacios saludables } \\
\text { - Establecimientos de educación } \\
\text { - Centros de trabajo } \\
\text { - Comunas y comunidades }\end{array}$ \\
\hline & & $\begin{array}{l}\text { Temáticas } \\
\text { - Tabaquismo } \\
\text { - Obesidad } \\
\text { - Sedentarismo }\end{array}$ \\
\hline
\end{tabular}


El trabajo de este grupo consistió en confeccionar mensajes claros y consensuados con la ayuda de especialistas en educación física de todo el país, mediante reuniones de expertos que examinaron los conceptos de salud, calidad de la vida, promoción de la salud y otros temas relacionados con la actividad física y el ejercicio. Posteriormente se elaboraron las recomendaciones con asesoramiento de consultores internacionales. Esas recomendaciones fueron validadas en cuatro regiones del país por 20 grupos focales en los que participaron profesionales de las ciencias sociales (antropólogos, sociólogos y psicólogos).

A continuación se aplicó en las instituciones y organizaciones relacionadas con el tema una encuesta de validación que incluía los mensajes validados por los grupos focales, con el fin de conocer su opinión y solicitar su apoyo para su posterior difusión. Con los resultados de esta validación se elaboraron seis mensajes definitivos, cada uno con su diseño gráfico correspondiente, que constituyeron la base de todas las campañas mediáticas sobre actividad física. Los mensajes fueron: "Camine al menos 30 minutos diarios"; "Realice ejercicios de estiramiento"; "Prefiera recrearse activamente"; "Realice pausas activas en el trabajo"; "Realice ejercicios 30 minutos 3 veces por semana y adecue la intensidad (alta, media, baja) a su ritmo personal".

Estrategias educativas y formación de recursos humanos. En 1999 VIDA CHILE inició el trabajo de promoción de la salud en los establecimientos de educación a través de su comité técnico de escuelas promotoras de la salud, integrado por los Ministerios de Salud y de Educación, la Junta Nacional de Auxilio Escolar y Becas, la Junta Nacional de Jardines Infantiles, la Fundación Integra y la Organización Panamericana de la Salud. Este trabajo abarcó intervenciones relacionadas con la actividad física, la alimentación, la salud bucal y el medio ambiente; la lucha contra el tabaquismo, el alcoholismo y el consumo de drogas; y algunos elementos psicosociales, tales como las habilidades para la vida, la afectividad, la sexualidad, las relaciones interpersonales y la participación social. $\mathrm{Al}$ año siguiente se adoptó un sistema de acreditación técnica basado en criterios y requisitos mínimos que ha permitido evaluar la marcha de este proceso en 607 establecimientos de educación acreditados como promotores de la salud. De ellos, $96 \%$ trabajan para mejorar la actividad física en los escolares y contribuir a reducir el sedentarismo en el país. Casi la mitad de estas escuelas (49\%) cuentan con un profesor capacitado en esta materia y $47 \%$ desarrollan actividades físicas tres veces a la semana, con lo que promueven la práctica de los deportes y el desarrollo de actividades recreativas entre los alumnos. De las escuelas acreditadas, $44 \%$ invirtieron en equipamiento deportivo y en el mejoramiento del espacio físico, y un tercio aumentó el tiempo curricular destinado a la educación física. La acreditación se realiza localmente y mide la programación de actividades, la participación de los diferentes integrantes de la comunidad educativa y las actividades de promoción organizadas, según su tipo y la cobertura alcanzada. Como resultado de este análisis, las instituciones son certificadas por las autoridades de educación y salud.

El aumento de las horas de actividad física curricular y extracurricular durante la jornada escolar forma parte de la reforma educacional que lleva adelante el Ministerio de Educación. En cuanto a la formación de recursos humanos en actividad física, este ministerio suscribió un convenio con el Instituto Nacional de Deportes a fin de apoyar el plan nacional de actividad física y deporte para escolares, que incentiva la práctica de la actividad física en las escuelas, mediante la capacitación de recursos humanos, la asesoría técnica y la entrega de material didáctico. Hasta el presente se ha capacitado a 2809 docentes en 70 cursos impartidos en el país.

Existe un acuerdo de colaboración entre Chile y Canadá para la promoción de la salud que cuenta con un programa de transferencia de tecnología canadiense. En el marco de este acuerdo se imparten talleres a los equipos locales encargados de echar a andar los programas de actividad física, alimentación y de lucha contra el tabaquismo (23). Mediante este convenio y a escala de proyectos pilotos, el Ministerio de Salud, junto con el Ministerio de Educación, el Instituto Nacional de Deportes y las universidades del país, han validado y adaptado a la realidad chilena el programa denominado Desafío Canadiense para la Vida Activa. Este programa — dirigido a niñas, niños y jóvenes de 6 a 18 años-contiene metodologías y módulos específicos que deben aplicarse en las escuelas (inserciones curriculares en educación básica y media) y en la comunidad. La validación realizada entre los años 2000 y 2001 se aplicó a 13 proyectos pilotos en el que participaron 1200 personas de cuatro regiones del país (I, III, VIII y X). La versión definitiva del programa les fue entregada a las autoridades $(24,25)$ y se comenzó a aplicar de forma experimental con el fin de evaluar su impacto.

Actividad física en la atención primaria de salud y en los lugares de trabajo. El Ministerio de Salud, mediante el convenio No. 1140 del 24 de julio de 2001 con el Instituto Nacional de Deportes, incorporó programas de actividad física para embarazadas y enfermos crónicos en los lugares de atención primaria de salud. Consistían en sesiones colectivas de ejercicios tres veces a la semana durante tres 
meses. Hasta el año 2001 se habían realizado 684 talleres con diferentes grupos. Esta experiencia ha permitido reorientar los servicios hacia la promoción de la salud y elaborar propuestas para la reforma del sistema de salud.

Se inició el programa de actividad física en las empresas, tanto públicas como privadas, mediante pausas con ejercicios en el puesto de trabajo. Las empresas asumieron el compromiso de autorizar la práctica deportiva y de estimular la organización de talleres de actividad física mediante incentivos directos, tales como subsidios o premios.

Recuperación de espacios públicos para la actividad física y la recreación. En el año 2001 se creó el programa Plazas VIDA CHILE, para contribuir a la recuperación de espacios públicos mediante la habilitación de plazas, parques, playas y otras áreas aptas para la realización de actividades físicas, ya sean recreativas, deportivas, de educación nutricional y ambiental, o actividades comunitarias de carácter artístico o cultural. Estas "tardes y mañanas entretenidas", abiertas a todos los ciudadanos, se realizan periódicamente y a ellas concurren familias, organizaciones sociales y comunitarias, diferentes asociaciones deportivas y entidades privadas.

Este programa funciona en la mayoría de las comunas del país. La meta propuesta para el año 2010 es que todas las comunas tengan al menos una Plaza VIDA CHILE. Al mismo tiempo, los municipios realizan periódicamente actividades de promoción comunitarias (maratones, recorridos en bicicleta, caminatas, campamentos y otras), acciones educativas de grupo (talleres con niños, padres, trabajadores, funcionarios, profesores de ejercicios aeróbicos, basquetbol, baile, yoga y otras), y actividades para la formación y capacitación de monitores. Para esto se utilizan lugares comunitarios, centros de salud y educación y recintos deportivos.

Campañas mediáticas. Desde 1999, VIDA CHILE y el Ministerio de Salud realizan campañas mediáticas de promoción de la salud destinadas a incentivar la vida activa y la alimentación saludable bajo el lema "Construyendo un país más saludable". Para esto se han utilizado "cuñas" radiales en emisoras nacionales y la prensa nacional. A escala regional se han empleado estrategias de mercadeo directo y medios de comunicación regionales (televisión y otros), mientras que a escala comunitaria se han usado eventos y medios locales (radio comunitaria, diarios locales e impresos). La evaluación de la primera de estas campañas mediante una prueba que medía la fijación de los mensajes en una muestra de la población tuvo resultados positivos (retención de los mensajes de $60 \%$ ). En las campañas siguientes se ha continuado registrando el número de elemen- tos promocionales distribuidos y los beneficiarios alcanzados.

Se han realizado también dos campañas mediáticas. La primera la llevó a cabo el Instituto Nacional de Deportes bajo el lema "Consume deporte ... la nueva meta de Chile" y con el argumento mediático "En Chile no existen excusas para no hacer deporte o actividad física". La segunda, llevada a cabo por el Ministerio de Salud con el mensaje central "Tu salud gana", utilizó como medio principal la vía pública de centros urbanos con los mensajes mediáticos "Deja los tacos ¡Ponte las zapatillas!”, “Levántate y anda!", “No ahorres, igasta calorías!" y "Baja calorías, sube las escaleras". Posteriormente, se han priorizado en la campaña mediática los mensajes de las guías de vida activa para la población chilena, especialmente el mensaje "Camina al menos 30 minutos diarios".

Investigación. Existen numerosos proyectos de investigación sobre la actividad física en los que participan los Ministerios de Educación y de Salud, el Instituto Nacional de Deportes y las universidades. Entre ellos existen proyectos dirigidos a la educación preescolar con el fin de validar las intervenciones en nutrición y actividad física realizadas en párvulos. Otros proyectos dirigidos a la educación básica buscan establecer estrategias de promoción de la salud que integren la alimentación, la actividad física, el medio ambiente y la lucha contra el tabaquismo. Hay también proyectos orientados a identificar indicadores que permitan medir el impacto de las intervenciones realizadas. Los resultados de estas investigaciones contribuirán en gran medida a la elaboración de políticas y al desarrollo de la estrategia de acreditar escuelas promotoras de la salud.

Regulación y legislación. La Ley 19.712, del 9 de febrero de 2001, consagra la responsabilidad del Estado en todo lo concerniente a la actividad física mediante la creación del Instituto Nacional de Deportes, con rango de subsecretaría. Su misión es crear las condiciones necesarias que faciliten, fomenten y apoyen la práctica de actividades físicas y deportivas de forma sistemática, y lograr con ello una vida de mejor calidad para toda la población. Este servicio público, relacionado con el Poder Ejecutivo por mediación de la Secretaría General de Gobierno, desempeña sus funciones mediante una red integrada por un consejo nacional, una dirección nacional y consejos y direcciones regionales.

Esta ley, conocida como la Ley del Deporte, ha sido ampliada por la Ley 19.787, de febrero de 2002 , con disposiciones especiales para fomentar el deporte mediante subsidios, la exención tributaria a las donaciones que se realicen con estos fines, la ampliación de la infraestructura deportiva y los me- 
canismos de concesión, así como para regular las organizaciones deportivas que se creen con este fin.

Para incentivar la actividad física hay que abordar dos áreas clave: la primera está dirigida al desarrollo normativo-curricular en el sistema educacional, con el fin de aumentar el número de horas de actividad física durante la educación preescolar, básica y media. La segunda, que está relacionada con las políticas de desarrollo urbano, se encamina a favorecer la creación de espacios públicos que puedan usarse para la vida activa - como parques y áreas verdes-, mejorar la calidad de las viviendas, dotar a las comunidades con medios para la recreación y el deporte - como vías especiales para bicicletas-y otros proyectos de obras públicas.

\section{RESULTADOS Y DIFICULTADES}

A pesar del corto tiempo transcurrido desde que se inició la política actual dirigida a promover una vida activa y la práctica de los deportes y ejercicios en la población en general, se han obtenido resultados alentadores y se ha logrado el apoyo de organizaciones sociales, así como de grupos técnicos, gubernamentales y académicos. Además, la población en general se ha incorporado a esta iniciativa.

La política de promoción de la actividad física se inscribe dentro de la política general de promoción de salud del país y le aporta un carácter más integral, gracias a sus diversas estrategias dirigidas a lograr una alimentación saludable, disminuir el hábito de fumar, proteger los elementos psicosociales y ambientales de la población relacionados con la actividad física y construir espacios saludables. Sin embargo, debido a la magnitud del problema en Chile, las complejidades que implica el cambio de hábitos en la población y los obstáculos que conlleva todo proceso de implementación intersectorial y participativo, no se pueden esperar cambios sustantivos a corto plazo.

Los principales obstáculos encontrados son el predominio de la cultura sectorial y biomédica, la existencia de programas públicos fragmentados, los escasos canales de cooperación y participación de esos programas, la insuficiencia de recursos presupuestarios y las bajas coberturas alcanzadas hasta el momento. El hecho de que en Chile se haya tomado la decisión política de convertir la promoción de la salud en política de estado - de manera que sea continua, trascienda a los gobiernos de turno, tenga cobertura nacional, goce de apoyo financiero y cuente con un sistema de regulaciones- no garantiza el éxito y la mejoría de los indicadores. Por esa razón se trabaja para acometer este cambio cultural y elaborar políticas saludables a largo plazo. Se ha comenzado por el trabajo con los niños, especial- mente en los jardines infantiles y escuelas promotoras de la salud, para inculcarles desde pequeños el hábito de una vida activa. En los otros grupos de edad se trabaja en la aplicación de las guías para una vida activa, con el fin de modificar los hábitos sedentarios de la población reflejados en las encuestas.

A la vez, con el fin de facilitar esta transición, es necesario franquear las barreras que se han ido creando con el crecimiento económico y la excesiva urbanización del país. Para ello es necesario ampliar el número de lugares aptos para la práctica de actividades físicas y promover el establecimiento de regulaciones adecuadas y de incentivos que estimulen la actividad física en el ámbito laboral, recreativo, familiar y otros.

\section{CONCLUSIONES}

La existencia de una política general de promoción de la salud — respaldada por importantes instituciones organizadas en VIDA CHILE-, el apoyo financiero y las regulaciones gubernamentales, así como la incorporación de grupos académicos comprometidos con este proceso de transición permiten avizorar el logro de los objetivos establecidos de pasar a una vida más activa y mejorar el estado de salud de la población en Chile.

\section{SYNOPSIS}

\section{Promoting health and physical activity in Chile: a policy priority}

This piece describes and analyzes the situation with respect to physical activity in Chile and the policies and strategies that have been developed in order to combat sedentary lifestyles. The level of sedentary lifestyles in the Chilean population is very high, with $91 \%$ performing less than 30 minutes of physical activity three times per week. This predicament has worsened as a result of increased urbanization and economic growth over the past decade, with a resulting increase in the number of cars and televisions, along with a rise in the number of hours that workers are on the job each day. In order to deal with this situation a policy to develop physical activity was implemented as a part of the country's general health promotion policy. This policy is overseen by the CHILE LIFE Council (Consejo VIDA CHILE), which is made up of 28 Chilean institutions. The policy encompasses various strategies, such as preparing guides to active living for the Chilean population, conducting educational activities and human resources training for kindergartens and other schools, establishing regulatory measures, performing research, carrying out media campaigns, reclaiming public spaces for recreation, and creating incentives for physical activity in the workplace. These strategies have been well received by the general population, academicians, professionals, and technicians. This makes it possible to foresee achieving in the medium and long term the objectives that have been set: more active lives and a better state of health for the Chilean population. 


\section{REFERENCIAS}

1. Vio F, Albala C. Nutrition policy in the Chilean transition. Pub Health Nutr 2000;3(1):49-55.

2. Albala C, Vio F, Kain J, Uauy R. Nutrition transition in Chile: determinants and consequences. Pub Health Nutr 2002;5(1A):123-128

3. Instituto Nacional de Estadísticas. Censo 2002. Chile: INE; 2002.

4. Vio F, Albala C, Crovetto M. Promoción de salud en la transición económica de Chile. Rev Chil Nutr 2000;(27):21-29.

5. Consejo Nacional para el Control de Estupefacientes. Cuarto Estudio Nacional de Consumo de Drogas en Chile: informe ejecutivo. Santiago, Chile: CONACE; 2002.

6. Prentice AM, Jebb SA. Obesity in Britain: gluttony or sloth? Br Med J 1995; 311:437-439.

7. Barlow CE, Kohl HW, Gibbons LW Blair SN. Physical fitness, mortality and obesity. Int J Obes 1995;19:S41-S44.

8. Olivares S, Albala C, García F, Jofré I. Publicidad televisiva y preferencias de alimentos en escolares de la Región Metropolitana. Rev Med Chile 1999;127: 791-799.

9. Berríos X, Jadue L, Zenteno J, Ross MI Rodríguez H. Prevalencia de factores de riesgo de enfermedades crónicas: un estudio poblacional en la Región Metropolitana de Santiago, Chile, 1986-1987. Rev Med Chile 1990;118:597-604.
10. Berríos X. Factores de riesgo en enfermedades crónicas de adultos. Un ejemplo de investigación epidemiológica. Bol Esc Med Univ Cat Chile 1994;23: 73-89.

11. Jadue L, Vega J, Escomar MC, Delgado I, Garrido C, Lastra P, et al. Factores de riesgo para enfermedades crónicas no transmisibles: métodos y resultados de la encuesta CARMEN. Rev Med Chile 1999;127:1004-1013

12. Chile, Ministerio de Planificación y Cooperación, Instituto Nacional de Deportes. Encuesta de Caracterización Socioeconómica Nacional (CASEN) 2000. Sector Deportes. Santiago, Chile: IND; 2001.

13. Chile, Ministerio de Salud. Encuesta de Calidad de Vida y Salud. Chile 2000. Santiago, Chile: MINSAL; 2001.

14. Chile, Ministerio de Salud. Plan Nacional de Promoción de la Salud. Santiago, Chile: MINSAL; 1999.

15. Salinas J. Health promotion in Chile: an evaluation of a national plan implementation. Prom Educ 2000;7:13-16.

16. Consejo Nacional para la Promoción de Salud VIDA CHILE. Plan estratégico 2001-2006. Metas intersectoriales al 2006. Santiago, Chile: MINSAL; 2000.

17. Ministerio de Salud. Planes comunales de promoción de la salud 2001. Santiago, Chile: MINSAL; 2001.
18. Acta de Huechuraba "Por un país más saludable". I Congreso de Promoción de la Salud, 24-27 de agosto de 1999, Santiago, Chile.

19. Consejo Nacional para la Promoción de la Salud VIDA CHILE. Cuenta pública 2001. Santiago, Chile: MINSAL-Impresos La Nación; 2002.

20. Chile, Ministerio de Salud. Objetivos sanitarios para Chile 2000-2010. Santiago, Chile: MINSAL; 2001

21. Salinas J, Vio F. Promoción de la salud en Chile. Rev Chil Nutr 2002;29:164-173.

22. Rosso P, Mardones F. Nutrición en el embarazo. En: Pérez A, Donoso E, eds. Obstetricia. 3. a ed. Santiago, Chile: Mediterráneo; 1999. Pp. 233-244.

23. Chile, Ministerio de Salud. Manual de apoyo a programas de promoción de la salud en alimentación, actividad física y tabaco. Santiago, Chile: MINSAL; 2001.

24. Consejo VIDA CHILE, Ministerio de Salud. El desafío chileno para una vida activa. [Serie de 4 manuales educativos y material de apoyo]. Santiago, Chile: MINSAL; 2001.

25. Salamo P, Salinas J, Pezoa S, Grunpeter $\mathrm{H}$, Jackson $\mathrm{S}$. The adaptation of a Canadian active living program in Chile. Toronto: Health Ministry, University of Toronto; 2002

\section{Call for Papers \\ II International Congress of Dengue and Yellow Fever}

Papers are being sought for symposium and poster presentations at the II International Congress of Dengue and Yellow Fever, which will be held in Havana, Cuba, 31 May to 3 June 2004.

Topics to be discussed at the Congress will include: clinical management; global epidemiology; vaccine initiatives and challenges; virology, diagnosis, and molecular biology; emergency plans; immunopathology and physiopathology; vector biology; dengue and society, including inequity and globalization; ecosystem approaches to dengue prevention; genetics and dengue; priorities for dengue research; vector control; behavior change; and yellow fever and other arboviruses.

The deadline for submitting an abstract for a proposed presentation is 1 April 2004. Authors will be notified by 20 April if their proposal has been accepted. Presentation abstracts should be sent by e-mail to "congreso.dengue@infomed.sld.cu," using the format specified in the Web site for the Congress.

\section{Information}

II International Congress of Dengue and Yellow Fever Internet: http://www.cidfa2004.sld.cu 